\title{
Simulation of a Luneburg Lens using a broadband Multilevel Fast Multipole Algorithm
}

\author{
Bart Michiels, ${ }^{1}$ Ignace Bogaert, ${ }^{1}$ Jan Fostier, ${ }^{1}$ Joris Peeters, ${ }^{1}$ and Daniël De Zutter ${ }^{1}$ \\ Received 30 August 2010; revised 2 December 2010; accepted 22 December 2010; published 8 March 2011. \\ [1] In this paper, the full wave simulation of a 2-D Luneburg lens is reported, using the \\ Multilevel Fast Multipole Algorithm (MLFMA). To stabilize the MLFMA at low \\ frequencies, it is augmented with a normalized plane-wave method, yielding a fully \\ broadband solver. To test the proposed method, the Luneburg lens is partitioned into \\ concentric shells with a constant permittivity, resulting in a complex simulation target \\ that consists of multiple embedded dielectric objects. The numerical results are \\ in good agreement with the analytical solutions for both the continuous and \\ discretized lens.
}

Citation: $\quad$ Michiels, B., I. Bogaert, J. Fostier, J. Peeters, and D. De Zutter (2011), Simulation of a Luneburg Lens using a broadband Multilevel Fast Multipole Algorithm, Radio Sci., 46, RS2003, doi:10.1029/2010RS004510.

\section{Introduction}

[2] Due to the growing interest in technology based on electromagnetics, such as wireless communication and photonics, it is important to be able to solve Maxwell's equations as quickly and as precisely as possible. When considering piecewise homogeneous media and perfect or imperfect conductors, one of the most popular and efficient simulation methods is the use of boundary integral equations and to discretize them using the method of moments (MoM) [Harrington, 1968]. In the MoM, the boundaries of the objects are divided into segments and for each segment the fields are expressed as a linear combination of basis functions. In this paper the Poggio-Miller-Chang-Harrington-Wu-Tsai (PMCHWT) formulation for the boundary integral equations is used, which yields accurate solutions, but is ill conditioned [Kolundzija and Djordjevic, 2002]. Applying the MoM leads to a set of linear equations for which the system matrix is a dense matrix. A direct solution of this set requires $\mathcal{O}\left(N^{3}\right)$ operations, with $N$ the number of unknowns, which becomes unfeasible for large $N$. By solving the set of equations using Krylov-based, iterative methods, the complexity can be reduced to $\mathcal{O}\left(P N^{2}\right)$, as each of the $P$ iterations requires the evaluation of matrix-vector products. If the problem is well conditioned then $P$ is much

\footnotetext{
${ }^{1}$ Department of Information Technology, Ghent University, Ghent, Belgium.

Copyright 2011 by the American Geophysical Union. 0048-6604/11/2010RS004510
}

smaller than $N$. A further reduction of the complexity can be achieved by applying the Multilevel Fast Multipole Algorithm (MLFMA) [Chew et al., 2001]. The MLFMA reduces the complexity of the matrix-vector multiplication from $\mathcal{O}\left(N^{2}\right)$ to $\mathcal{O}(N \log N)$, allowing to solve problems with a large number of unknowns.

[3] If the number of unknowns $N$ is large, the computational requirements exceed the capabilities of a single processor and a parallel MLFMA has to be invoked. A partitioning scheme for a scalable parallel MLFMA has been presented by Fostier and Olyslager [2008] and Ergül and Gürel [2009a].

[4] All the previously mentioned methods and algorithms are implemented in Nero2d, which is open source and can be downloaded free of charge at http://www. openfmm.net.

[5] This paper focuses on the simulation of the Luneburg lens [Luneburg, 1944; Kay, 1959; Bogaert et al., 2007; Parfitt et al., 2000] involving many unknowns, but at the same time exhibiting a complex geometry. Such problems require an MLFMA approach that remains stable and accurate at low frequencies, but at the same time remains truly broadband. Indeed, at the considered frequencies, the size of the MLFMA boxes on some of the lower levels is small with respect to the wavelength, whereas, at the higher levels, box sizes are comparable to the wavelength.

[6] In section 2, a very short recapitulation of the MLFMA for 2-D is given, indicating that the recently developed normalized plane-wave method (NPWM) [Bogaert et al., 2006] is a robust way to solve the so-called low-frequency breakdown of the classical MLFMA. 
[7] Section 3 considers the 2-D Luneburg lens geometry. The permittivity of this lens varies continuously as a function of its radius and focuses an incident plane wave into a single point on its surface. For the 2-D case, the solution of the problem can be written down analytically. As our 2-D MLFMA method can only handle objects with a constant permittivity and permeability, the Luneburg lens is divided into shells with constant material parameters, approximating the continuous lens. This results in a geometry where objects are embedded into other objects. An analytical solution for this discretized version of the lens is also available. By comparing the available analytical solutions and the numerical results from our NPWM-MLFMA, the validity of our numerical technique can be put to the test. A very similar approach to simulate a 3-D Luneburg lens has been presented by Carayol and Stève [2010]. Many other approaches to model complex geometries exist, [e.g., Jordan et al., 2009]. Finally, section 4 presents some conclusions.

\section{A Low-Frequency Stable MLFMA}

[8] In 2-D problems the MLFMA is based on the following expansion of the Green's function [Chew et al., 2001]:

$$
\begin{gathered}
H_{0}^{(2)}(k \rho) \simeq \frac{1}{2 \pi} \int_{0}^{2 \pi} e^{j \vec{k} \cdot \vec{\rho}_{a}} T\left(\vec{\rho}_{T}\right) e^{j \vec{k} \cdot \vec{\rho}_{d}} d \phi \\
T\left(\vec{\rho}_{T}\right)=\sum_{n=-Q}^{Q} j^{n} H_{n}^{(2)}\left(k \rho_{T}\right) e^{j n\left(\phi_{T}-\phi\right)},
\end{gathered}
$$

with $\vec{\rho}=\vec{\rho}_{a}+\vec{\rho}_{T}+\vec{\rho}_{d}$, $k$ the wave number, $\vec{\rho}_{a}$ the aggregation vector, $\vec{\rho}_{T}$ the translation vector, $\vec{\rho}_{d}$ the desaggregation vector and $H_{n}^{(2)}(z)$ the Hankel function of the second kind and the $n$th order.

[9] For low frequencies the order $n$ in (1b) can become larger than the argument $k \rho_{T}$. For these terms the Hankel function increases rapidly, leading to numerical instabilities. Bogaert et al. [2006] solved this instability by the introduction of the Normalized Plane Wave Method (NPWM). In the NPWM the translation operator $T\left(\vec{\rho}_{T}\right)$ is split into two parts: $T^{ \pm}\left(\vec{\rho}_{T}\right)$. Because the integrand is holomorphic and has a periodicity of $2 \pi$, the integration path can be shifted over a distance $\mp j \chi$. The strong increase of the Hankel functions is now compensated by a factor $e^{-|n| \chi}$, which makes the addition theorem of (1a) and (1b) numerically stable. A first validation of the NPWM, hybridized with the MLFMA, is provided by Michiels et al. [2009]. In three dimensions, the addition theorem likewise suffers from a low-frequency breakdown and a similar stabilization technique, the so-called Nondirective Stable Plane Wave MLFMA (NSPWMLFMA), is analyzed by Bogaert and Olyslager [2009a] and Peeters et al. [2008]. Other techniques to stabilize the low-frequency breakdown also exist, for instance the techniques presented by Bogaert and Olyslager [2009b] for two dimensions and Bogaert et al. [2008] for three dimensions. Instead of using the addition theorem of (1a) and (1b), also fast multipole methods with nondiagonal translation operators can be employed, as for example done by Greengard and Rokhlin [1987].

[10] All numerical results presented in the following are based on the method first described by Bogaert et al. [2006]. The used basis functions are piecewise linear for the longitudinal field components $E_{z}$ and $H_{z}$ and piecewise constant for the tangential field components $E_{t}$ and $H_{t}$.

\section{The Luneburg Lens}

[11] To thoroughly test our boundary integral equation solver, a Luneburg lens will be simulated. A 2-D Luneburg lens is an infinitely long cylinder with circular cross section and its refractive index depends on the radial coordinate $\rho$, such that

$$
\epsilon(\rho)=\epsilon_{0}\left(2-\frac{\rho^{2}}{R^{2}}\right) \quad \rho<R,
$$

where $R$ is the radius of the lens.

[12] When a plane wave impinges perpendicularly to the cylinder axis, the incident field is focused in a point on the surface of the cylinder. The line connecting the focal point to the center of the cross section is parallel to the direction of incidence.

[13] As our boundary integral equation solver can only handle objects with a constant permittivity, the continuous permittivity profile (2) is approximated by means of concentric shells with a constant permittivity. Hence, solving the Luneburg lens requires the correct handling of dielectric objects embedded in other dielectrics. Also, when a large number of shells or a very dense discretization is used, a broadband MLMFA is required to solve this problem efficiently, making the Luneburg lens a rather challenging problem. However, as a test case it is particularly suited to validate the solver, since analytical solutions exist for both the continuous and piecewise constant Luneburg lens. This allows us to split the total error on the numerical solution into two parts: the error made by approximating the continuous permittivity profile with a piecewise constant one, and the error introduced by our solver. This in turn will allow a thorough validation of our solver. 


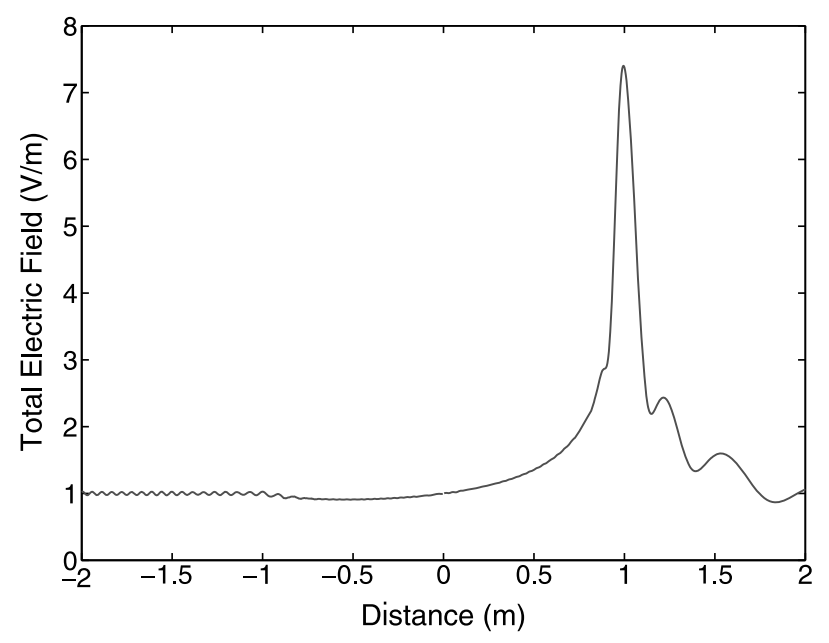

Figure 1a. Amplitude of the total electric field as a function of the spatial coordinate along the direction of incidence. The focal point of the Luneburg lens is $E_{0}=$ $1 \frac{\mathrm{V}}{\mathrm{m}}, R=1 \mathrm{~m}$.

\subsection{Analytical Solutions}

[14] In this paragraph, the analytical solutions for the continuous and piecewise constant Luneburg lens will be briefly derived. Then these two analytical results will be compared to the results obtained using our boundary integral equation solver.

[15] The excitation is assumed to be a TM-polarized plane wave ( $\mathbf{E}^{i n}=E_{z}^{i n} \mathbf{u}_{z}$, i.e., parallel to the cylinder axis). The analytical solution for the continuous Luneburg lens is obtained using separation of variables

$$
\begin{aligned}
& E_{z}^{i n}=E_{0} e^{-j k_{0} \rho \cos \phi}, \\
& =\sum_{n=0}^{+\infty} E_{0}(-j)^{n} \nu_{n} J_{n}\left(k_{0} \rho\right) \cos (n \phi) \quad \rho>R, \\
& E_{z}^{s c}=\sum_{n=0}^{+\infty} E_{n}^{s c} \nu_{n} H_{n}^{(2)}\left(k_{0} \rho\right) \cos (n \phi) \quad \rho>R, \\
& E_{z}=\sum_{n=0}^{+\infty} E_{n} \nu_{n} \frac{1}{\rho} W M \\
& \cdot\left(\frac{k_{0} R}{2}, \frac{n}{2}, \frac{k_{0} \rho^{2}}{R}\right) \cos (n \phi) \quad \rho<R, \\
& \nu_{n}=2-\delta_{n 0},
\end{aligned}
$$

with $J_{n}(z)$ the Bessel function of the first kind and $n$th order. $W M(\kappa, \nu, z)$ is the Whittaker function, denoted by Abramowitz and Stegun [1965, pp. 505-507] by $M_{\kappa, \nu}(z)$. Furthermore, $\delta_{n 0}$ equals 1 for $n=0$ and zero for all other $n$, while $k_{0}$ is the free space wave number and the superscript "sc" stands for the scattered field.

[16] The complex coefficients $E_{n}^{s c}$ and $E_{n}$ can be found by enforcing the proper boundary conditions at $\rho=R$. Using Abramowitz and Stegun [1965, formulas 9.1.27 and 13.4.32], the continuity of the tangential electric and magnetic field leads to

$$
\left[\begin{array}{ll}
Z_{11} & Z_{12} \\
Z_{21} & Z_{22}
\end{array}\right]\left[\begin{array}{c}
E_{n}^{s c} \\
E_{n}
\end{array}\right]=\left[\begin{array}{l}
B_{1} \\
B_{2}
\end{array}\right]
$$

with

$$
\begin{gathered}
B_{1}=E_{0}(-j)^{n} J_{n}\left(k_{0} R\right), \\
B_{2}=E_{0}(-j) \frac{{ }^{n} \frac{k_{0}}{2}\left(J_{n-1}\left(k_{0} R\right)-J_{n+1}\left(k_{0} R\right)\right),}{Z_{11}=-H_{n}^{(2)}\left(k_{0} R\right),} \\
Z_{12}=\frac{1}{R} W M\left(\frac{k_{0} R}{2}, \frac{n}{2}, k_{0} R\right), \\
Z_{21}=-\frac{k_{0}}{2}\left(H_{n-1}^{(2)}\left(k_{0} R\right)-H_{n+1}^{(2)}\left(k_{0} R\right)\right), \\
\frac{1}{R^{2}}\left(\left(k_{0} R+n+1\right) W M\left(\frac{k_{0} R}{2}+1, \frac{n}{2}, k_{0} R\right)\right. \\
\left.-W M\left(\frac{k_{0} R}{2}, \frac{n}{2}, k_{0} R\right)\right) .
\end{gathered}
$$

[17] Figure 1a shows the amplitude of the total electric field along the direction of incidence for an incident wave with a wavelength $\lambda_{0}=R / 10$ in free space and for $R=$ $1 \mathrm{~m}$. In the focal point on the surface of the cylinder the field is high, which clearly shows that the field is focused in this point. Figure $1 \mathrm{~b}$ shows the phase of the total electric field over the cross section. When the plane wave impinges on the lens, the flat phase fronts are gradually bent toward the focal point.

[18] To obtain the analytical solution for the piecewise constant Luneburg lens, the Luneburg lens is first divided 


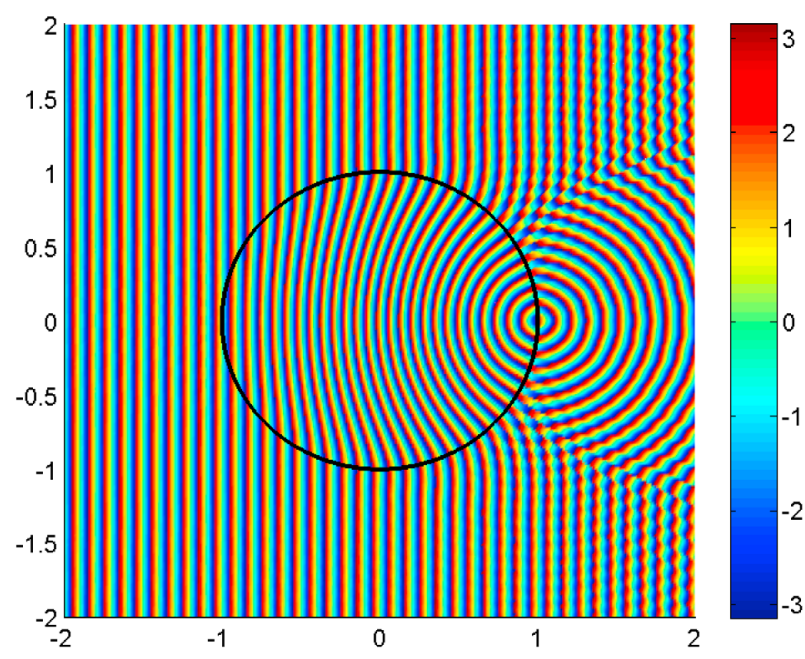

Figure 1b. Phase of the total electric field over the cross section. The flat phase fronts of the plane wave are gradually bent toward the focal point. The focal point of the Luneburg lens is $E_{0}=1 \frac{\mathrm{V}}{\mathrm{m}}, R=1 \mathrm{~m}$.

into $M$ shells. Each shell $i(i=1 \ldots M)$ is selected to have a radius $R_{i}$ and permittivity $\epsilon_{i}$ determined by

$$
\begin{gathered}
\int_{0}^{R_{1}} \epsilon(\rho) \rho d \rho=\int_{R_{1}}^{R_{2}} \epsilon(\rho) \rho d \rho=\ldots=\int_{R_{i-1}}^{R_{i}} \epsilon(\rho) \rho d \rho \\
=\ldots=\int_{R_{M-1}}^{R} \epsilon(\rho) \rho d \rho, \\
\int_{R_{i-1}}^{R_{i}} \epsilon(\rho) \rho d \rho=\epsilon_{i} \int_{R_{i-1}}^{R_{i}} \rho d \rho \\
\quad \forall i=1 \ldots M,
\end{gathered}
$$

with $M$ the number of shells. Of course, other criteria could be used to discretize the continuous lens.

[19] When the piecewise constant permittivity profile has been determined, the analytical solution is also obtained using separation of variables. The analytical expression for the electric field in each shell and for the scattered field outside the lens is given by

$$
\rho>R: \quad E_{z}^{s c}=\sum_{n=0}^{+\infty} \nu_{n} E_{n}^{s c} H_{n}^{(2)}\left(k_{0} \rho\right) \cos (n \phi),
$$

$$
\begin{aligned}
R_{i-1} & <\rho<R_{i}: \\
E_{z, i} & =\sum_{n=0}^{+\infty} \nu_{n}\left(E_{i, n}^{1} J_{n}\left(k_{i} \rho\right)+E_{i, n}^{2} Y_{n}\left(k_{i} \rho\right)\right) \cos (n \phi),
\end{aligned}
$$

$$
\rho<R_{1}: \quad E_{z, 1}=\sum_{n=0}^{+\infty} \nu_{n} E_{1, n}^{1} J_{n}\left(k_{1} \rho\right) \cos (n \phi),
$$

where $i$ varies from 2 to $M$ and $Y_{n}(z)$ is the Bessel function of the second kind and the $n$th order. The wave numbers of the respective shells are denoted by $k_{i}$.

[20] Imposing the boundary conditions, i.e., continuity of the tangential electric and magnetic field at each shell boundary, leads to a set of $2 M$ linear equations with $2 M$ unknowns, for each $n$, which allows the amplitudes $E_{n}^{s c}$, $E_{i, n}^{1}, E_{i, n}^{2}$ and $E_{1, n}^{1}$ to be determined.

\subsection{Results}

[21] Figures $2 \mathrm{a}$ and $2 \mathrm{~b}$ show the comparison between the three solutions: the analytical solution for the continuous Luneburg lens, the analytical solutions for the lens divided into shells and the numerical solution for the shell approximation using the broadband MLFMA-MoM solver. As the focal point of the Luneburg lens is the point of interest, the value of the electric field in this point is used for comparison. The relative error shown in Figures $2 \mathrm{a}$ and $2 \mathrm{~b}$ is defined as $\left|\frac{E_{z, \text { pipecewise }}}{E_{z, \text { continous }}}-1\right|$ and $\left|\frac{E_{z, \text { numerical }}}{E_{z, \text { piecewise }}}-1\right|$, respectively. The series in the analytical solutions (3a)-(3e) and (7a)-(7c) were truncated at 100 terms, thus ensuring convergence.

[22] Both the MLFMA precision and the iterative precision were set to $10^{-6}$. The former implies that the series in (1b) was truncated such that the error when applying the addition theorem of (1a) and (1b) is below

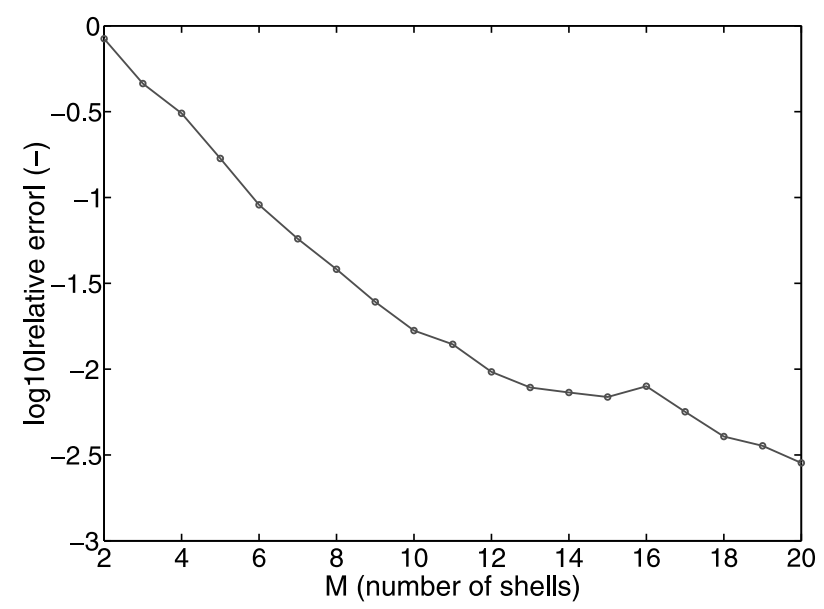

Figure 2a. Relative error of the analytical solution of the shells with respect to the analytical solution of the continuous Luneburg lens as a function of the number of shells. Comparison between the analytical solution for the Luneburg lens, the analytical solution for the lens divided in shells, and the numerical results. 


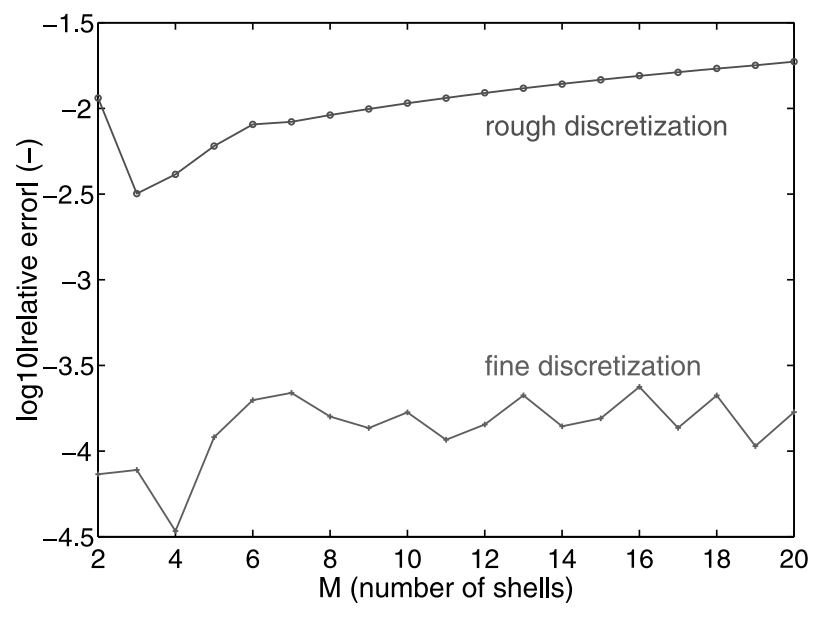

Figure 2b. Relative error of the numerical simulations (rough discretization is $\lambda_{0} / 10$, fine discretization is $\left.\lambda_{0} / 100\right)$ with respect to the analytical solution of the shells problem as a function of the number of shells. Comparison between the analytical solution for the Luneburg lens, the analytical solution for the lens divided in shells, and the numerical results.

$10^{-6}$. The latter means that the set of linear equations $\overline{\bar{Z}}$. $\bar{X}=\bar{B}$ is iteratively solved until $\|\bar{Z} \cdot \bar{X}-\bar{B}\|<10^{-6}\|\bar{B}\|$. The used integral equation formulation, the PMCHWT formulation, is ill conditioned [Ergül and Gürel, 2006], resulting in a slow convergence of the solution. To reduce the number of iterations, a block-diagonal preconditioner [Ergül and Gürel, 2009b] was used.

[23] Two different discretizations were used for the numerical simulations. For the first set of simulations the boundaries were discretized into segments of $\lambda_{0} / 10$, whereas for the second set of simulations the length of the segments was $\lambda_{0} / 100$. For a piecewise constant Luneburg lens with $M=20$ shells, this leads to 19506 and 194882 unknowns, respectively. The entire structure spans multiple wavelengths, whereas the individual segments are much smaller than the wavelength, therefore this is a challenging broadband problem. On two quad core AMD Opteron 2350 processors and a total of $32 \mathrm{~GB}$ of RAM, the simulations for $M=20$ discretized by $\lambda_{0} / 10$ and $\lambda_{0} / 100$ were solved in $1 \mathrm{~min} 3 \mathrm{~s}$ and $42 \mathrm{~min}$ $51 \mathrm{~s}$, respectively.

[24] As expected, Figure 2a shows that the Luneburg lens is more accurately approximated when the number of shells increases. Only for $M=16$ we notice a very small increase of the relative error. Figure $2 b$ displays the relative error between the numerical solution for the piecewise constant Luneburg lens as compared to its analytical counterpart. As we can see from Figure 2b, the relative error of the fine discretization is about a factor
100 better than the error of the rough discretization, which is in line with the expected convergence rates. For each discretization, the relative error remains more or less constant as a function of the number of shells, because each shell is divided into segments with a length of $\lambda_{0} / 10$ and $\lambda_{0} / 100$, respectively. The numerical results clearly show that our technique is capable of correctly handling broadband problems and objects embedded inside other objects allowing the simulation of a wide range of applications.

\section{Conclusion}

[25] In this paper electromagnetic scattering problems at a Luneburg lens were numerically solved by using boundary integral equations, discretized by means of the method of moments. The classical MLFMA was hybridized with the NPWM to allow the simulation of broadband problems. The numerical results for the Luneburg lens are in very good agreement with the analytical solutions. For an increasing number of shells in the discretized lens, the relative error between the analytical and numerical solution remains almost constant. This proves that our method is capable of handling such complex problems.

[26] Acknowledgment. This work was supported by a research grant of the Ghent University Research Council (BOF).

\section{References}

Abramowitz, M., and I. A. Stegun (1965), Handbook of Mathematical Functions with Formulas, Graphs and Mathematical Tables, Dover, New York.

Bogaert, I., and F. Olyslager (2009a), A low frequency stable plane wave addition theorem, J. Comput. Phys., 228 , 1000-1016.

Bogaert, I., and F. Olyslager (2009b), A broadband stable addition theorem for the two dimensional MLFMA, paper presented at Symposium on Antennas and Propagation, Inst. of Electr. and Electr. Eng., Charleston, S. C., 1-5 June.

Bogaert, I., D. Pissoort, and F. Olyslager (2006), A normalized plane wave method for 2-D Helmholtz problems, Microwave Opt. Technol. Lett., 48, 237-243.

Bogaert, I., L. Meert, and F. Olyslager (2007), Fast full-wave validation of a metamaterial Luneberg lens, paper presented at 2007 Symposium on Antennas and Propagation, Inst. of Electr. and Electr. Eng., Honolulu, 9-15 June.

Bogaert, I., J. Peeters, and F. Olyslager (2008), A nondirective plane wave MLFMA stable at low frequencies, IEEE Trans. Antennas Propag., 56, 3752-3767.

Carayol, Q., and H. Stève (2010), Computation of Luneburg lens scattering using multi-domain MLFMA, paper presented 
at National Radio Science Meeting, U.S. Natl. Comm., Int. Union of Radio Sci., Boulder, Colo., 5-8 Jan.

Chew, W. C., J. Jin, E. Michielssen, and J. Song (2001), Fast and Efficient Algorithms in Computational Electromagnetics, Artech House, Norwood, Mass.

Ergül, Ö., and L. Gürel (2006), Improving the accuracy of the magnetic field integral equation with the linear-linear basis functions, Radio Sci., 41, RS4004, doi:10.1029/2005RS003307.

Ergül, Ö., and L. Gürel (2009a), A hierarchical partitioning strategy for an efficient parallelization of the Multilevel Fast Multipole Algorithm, IEEE Trans. Antennas Propag., 57, $1740-1750$

Ergül, Ö., and L. Gürel (2009b), Efficient solution of the electric and magnetic current combined-field integral equation with the multilevel fast multipole algorithm and block-diagonal preconditioning, Radio Sci., 44, RS6001, doi:10.1029/2009RS004143.

Fostier, J., and F. Olyslager (2008), An asynchronous parallel MLFMA for scattering at multiple dielectric objects, IEEE Trans. Antennas Propag., 56, 2346-2355.

Greengard, L., and V. Rokhlin (1987), A fast algorithm for particle simulations, J. Comput. Phys., 73, 325-348.

Harrington, R. F. (1968), Field Computation by Moment Methods, Macmillan, New York.

Jordan, J., B. Sternberg, and S. Dvorak (2009), Development and validation of a low-frequency modeling code for high- moment transmitter rod antennas, Radio Sci., 44, RS6008, doi:10.1029/2008RS004010.

Kay, A. F. (1959), Spherically symmetric lenses, IEEE Trans. Antennas Propag., 7, 32-38.

Kolundzija, B., and A. Djordjevic (2002), Electromagnetic Modeling of Compsite Metallic and Dielectric Structures, Artech House, Norwood, Mass.

Luneburg, R. K. (1944), Mathematical Theory of Optics, Brown Univ., Providence, R. I.

Michiels, B., J. Fostier, J. Peeters, I. Bogaert, D. Pawlak, and F. Olyslager (2009), Fast full-wave scattering at extremely large and complex multiscale objects, paper presented at International Symposium on Antennas and Propagation, Inst. of Electr. and Electr. Eng., Charleston, S. C., 1-5 June.

Parfitt, A., J. Graeme, J. Kot and P. Hall (2000), A case for the Luneburg lens as the antenna element for the square kilometre array radio telescope, Radio Sci. Bull., 293, 32-37.

Peeters, J., I. Bogaert, J. Fostier, and F. Olyslager (2008), Fullwave 3-D simulations using the broadband NSPWMLFMA, paper presented at International Union of Radio Science General Assembly, Chicago, Ill., 7-16 Aug.

I. Bogaert, D. De Zutter, J. Fostier, B. Michiels, and J. Peeters, Department of Information Technology, Ghent University, SintPietersnieuwstr. 41, B-9000 Ghent, Belgium. (bart.michiels@ intec.ugent.be) 